\title{
75. PHOTOELECTRIC OBSERVATIONS OF MAGELLANIC CLOUD CEPHEIDS
}

\author{
S. C. B. Gascoigne \\ Mount Stromlo Observatory
}

This is a brief account of some photoelectric observations of Magellanic Cloud cepheids, begun in 1951 with Dr. G. E. Kron on the 30-inch reflector, and since continued there and on the 50- and 74-inch reflectors. The initial emphasis was on colour, the Cloud cepheids, as measured, being considerably bluer than those in the Galaxy. It is now known that this discrepancy is due mostly to reddening of the galactic cepheids, and interest has shifted rather to the differences in the period-luminosity relations in the two Clouds, as revealed by measurements made in the SMC by Arp (1960), and in the LMC by Woolley et al. (1962).

The work of these two groups was primarily photographic. They measured all the cepheids down to a limit of $V \simeq 17 \cdot 5$, within the areas defined by their plates, about a degree square in each case, the plates being calibrated photoelectrically.

TABLE 1

COMPARISON BETWEEN PHOTOGRAPHIC AND PHOTOELECTRIC

OBSERVATIONS

\begin{tabular}{|c|c|c|c|c|c|c|}
\hline \multirow{2}{*}{ Star } & \multicolumn{2}{|c|}{$\mathbf{P g}$} & \multicolumn{2}{|c|}{$\mathrm{Pe}$} & \multicolumn{2}{|c|}{$\mathrm{Pe}-\mathrm{Pg}$} \\
\hline & $\langle V\rangle$ & $<B>$ & $<V>$ & $<B>$ & $\langle V\rangle$ & $<B>$ \\
\hline 837 & $13 \cdot 09$ & $13 \cdot 98$ & $13 \cdot 30$ & $14 \cdot 19$ & $0 \cdot 21$ & $0 \cdot 21$ \\
\hline 886 & $13 \cdot 18$ & $13 \cdot 94$ & $13 \cdot 26$ & $14 \cdot 02$ & 0.08 & $0 \cdot 08$ \\
\hline HV 2251 & $13 \cdot 03$ & $13 \cdot 83$ & $13 \cdot 10$ & $13 \cdot 85$ & 0.07 & $0 \cdot 02$ \\
\hline HV 2294 & $(12 \cdot 51)$ & $(13 \cdot 29)$ & $12 \cdot 72$ & $13 \cdot 57$ & $0 \cdot 21$ & $0 \cdot 28$ \\
\hline
\end{tabular}

The present work, on the other hand, had to be confined to stars bright enough to be visible in the 30 -inch (later in the 50 - and 74 -inch telescopes), that is, to the brighter, longer-period cepheids. But because we were not limited to a particular part of either Cloud our coverage of these stars is fairly complete. In addition we have obtained light curves for three SMC cepheids of periods $4 \cdot 55,6 \cdot 29$, and $6 \cdot 65$ days respectively. Attempts to observe two cepheids of similar period in the LMC were defeated by the weather. In all, we have reasonably complete light curves for 14 SMC and 11 LMC cepheids. Eighteen of these stars have periods longer than 20 days, as opposed to a total of 12 observed by the photographic observers.

We have one star in common with Arp, and three with the Herstmonceux group. The comparison is made in Table 1. It shows reasonable agreement, bearing in mind that HV 837 and 2294 are both near the edges of the plates. Photoelectric results are, of course, not necessarily error-free, especially when made in crowded fields. Due attention was given to this point, however, and for the fainter stars both variable and comparison field were selected from 74-inch plates taken especially for the purpose. 
Figure $1(a)$ is a plot of $\langle V\rangle$ against $\log P$ for our 25 cepheids, and Figure $1(b)$ of $\langle B\rangle$ against $\log P$. Also plotted are the mean period-luminosity lines found for the SMC by Arp, and for the LMC by the Herstmonceux group (see Table 2). In the LMC the photoelectric results are systematically fainter than the Herstmonceux line, by a few tenths of a magnitude in each colour. For periods longer than 10 days this line is defined by five stars, only two of them not common to the present program. In this period range the 10 stars observed photoelectrically should therefore carry substantially more weight, a view strengthened by the surprisingly homo-
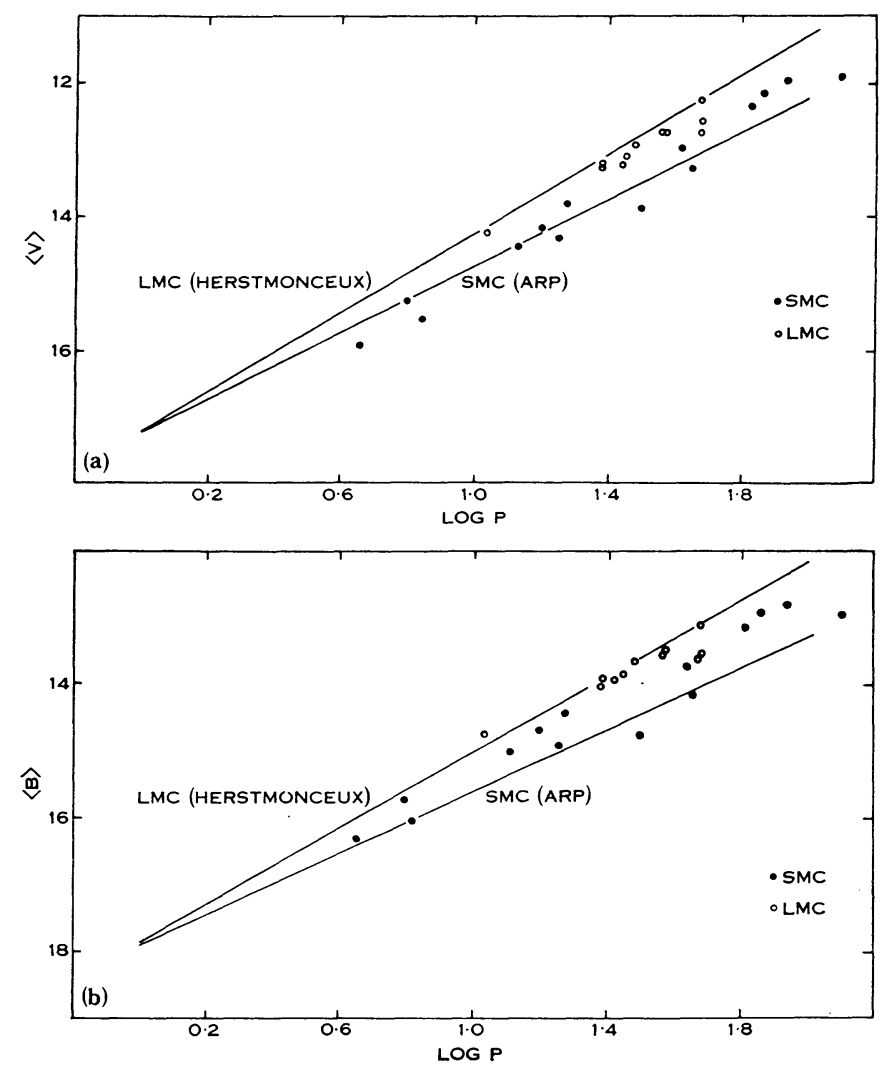

Fig. 1.-The mean magnitudes $(a)\langle V\rangle$ and $(b)\langle B\rangle$ plotted against $\log P$ for the cepheids on the present program. Also shown are the mean period-luminosity lines determined by Arp, and by Woolley et al. (Table 2).

geneous character of these stars - they show very little scatter in luminosity for their periods, and have similar large amplitudes in both light and colour. At present the best $P-L$ relation for the LMC is probably derived from a combination of Herstmonceux results for $P<10$ days, and of ours for $P>10$ days. Such a line has a slope of about $0 \cdot 15$ less than that of the Herstmonceux line (see Table 2).

In the SMC the situation is more complex. Our cepheids here show much more scatter in luminosity than in the LMC, and in the mean they are appreciably brighter than Arp's curve in $B$, though not in $V$ - in other words they are signifi- 
cantly bluer than Arp's cepheids. Also, they define $P-L$ relations appreciably steeper than Arp's. The situation is displayed in Table 2, where various $P-L$ lines are compared.

\section{TABLE 2}

PERIOD-LUMINOSITY RELATIONS

\begin{tabular}{l|c|c}
\multicolumn{1}{c|}{ Observations } & $<B>$ & $<V\rangle$ \\
\hline $\begin{array}{l}\text { Herstmonceux: } \\
13 \text { LMC stars of large amplitude }\end{array}$ & $17 \cdot 86-2 \cdot 85 \log P$ & $17 \cdot 22-2 \cdot 94 \log P$ \\
Arp: & $17 \cdot 74-2 \cdot 23 \log P$ & $17 \cdot 24-2 \cdot 48 \log P$ \\
$\quad 23$ SMC stars of large amplitude & $17 \cdot 90-2 \cdot 49 \log P$ & $17 \cdot 74-2 \cdot 87 \log P$ \\
Gascoigne: \\
$\quad 14$ SMC stars
\end{tabular}

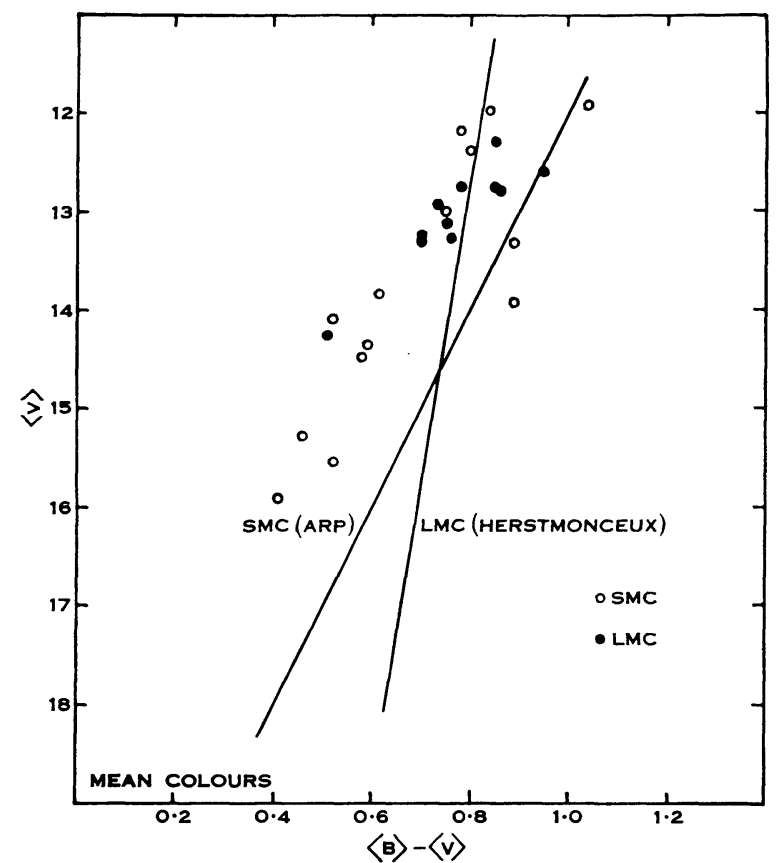

Fig. 2.-Mean colours $\langle B\rangle-\langle V\rangle$ plotted against $\langle V\rangle$ for all cepheids on our program. Also plotted are the mean lines determined for the SMC by Arp, and for the LMC by Woolley et al. No correction for reddening has been made to any of the data.

The addition of the present results has had the general effect of reducing the difference between the slopes of the $P-L$ lines for the two Clouds. At least two other lines of evidence (this volume, papers 66 and 72) suggest that the modulus of the LMC may be less than that of the SMC by from two- to perhaps as much as fourtenths of a magnitude. If this is so, we find, in the period range $20-50$ days, little 
difference between the luminosities of the cepheids in the two Clouds. The case for a difference between the Cloud period-luminosity relations rests then entirely on the photographic observations for cepheids of shorter period.

Returning to Table 2, Arp's $P-L$ relations lean heavily on shorter-period cepheids, ours on longer, and a detailed plot of the data shows that, at least in $V$, a non-linear $P-L$ relation would satisfy all results fairly well. However, the position cannot be regarded as satisfactory until the colour discrepancy has been cleared up. This disturbing situation is illustrated in Figure 2. Here the colours are plotted as observed, no allowance having been made for reddening. Excluding the possibility of photometric errors, which on this scale are unlikely, it appears either that all three members of our short-period sample are abnormal, or that there is a regional variation in the properties of the SMC cepheids, or that there is local absorption and reddening in that part of it studied by Arp. The last option seems to the writer less likely on a number of grounds, such as the low reddening shown in Arp's colour-magnitude diagrams for NGC 361 and 419, both just beyond his cepheid field; but it cannot be excluded, and the need for a cepheid program similar to Arp's in another part of the SMC is evident.

References

ARP, H. C. (1960).-A.J. $65: 404-44$.

Woolley, R. v. D. R. ET AL. (1962).-Royal Observatory Bull. No. 58.

\section{Discussion}

Eggen: In HD 1400 there appears to be some variation from cycle to cycle. Could it be a $\mathrm{W}$ Vir star? It is one of the three which gives a different slope to the colour-luminosity curve.

Gascoigne: It is also the lowest on the period-luminosity curve.

Woolley: There is a collection of Cape plates at Herstmonceux exposed on a second field in the LMC which has not yet been worked up. I hope that Dr. Gascoigne will join us in some of the work of reducing these plates when he visits Herstmonceux this year. I have the impression that it might be possible to reconcile the results in the LMC and SMC with a certain adjustment of the distances such as Dr. Gascoigne suggests, and I think that it would be unwise to speculate on possible differences between the two Clouds until further work is done.

Arp : Gascoigne's cepheid results agree extremely well with mine if you assume that there is $0.06 \mathrm{mag}=E_{B-V}$ more reddening in the NGC 371 field than that which I originally derived. This new reddening agrees well with that recently found by Feast, Thackeray, and Wesselink for this NGC 371 region.

I would also like to point out that because of the possible different spatial distributions of young and old cepheids that one reddening in a field may not apply to all cepheids in the field. If the long-period variables are differently reddened from the short, then the slope of the $P-L$ relation can be apparently changed from the true slope.

Eggen: This is quite true, but it would not make up the entire difference in colour. In each case the foreground absorption is the same.

Gascoigne: Arp studied the clusters NGC 361 and 419. In each field he found low reddening. Can there really be a blob of dust inside a circle which excludes these two clusters?

Feast : With reference to Dr. Gascoigne's last remark it should be pointed out that Dr. Arp and I apparently now agree that the reddening in the region of NGC 371 is about 0 m.05 greater than that originally adopted by Arp.

In Dr. Gascoigne's plot of cepheid colours against period, have these colours been corrected for interstellar reddening?

Gascoigne: No, but Arp's and Kraft's are. This means the position is worse than it looks. 